\title{
A Comparative Analysis of B2C Cross-border E-commerce Platforms: the AliExpress and the DHgate
}

\author{
Li Dehui \\ School of Economics and Management \\ South China Normal University \\ Guangzhou, China \\ lidehui@m.scnu.edu.cn
}

\begin{abstract}
As the most promising blue ocean industry, B2C cross-border e-commerce is growing rapidly with the support of government policies. This paper takes two of the country's biggest B2C cross-border e-commerce platforms as research objects, uses the method of comparative analysis to analyze them, aims to provide reference for building and enhancing the $\mathrm{B} 2 \mathrm{C}$ cross-border e-commerce platforms and businesses. The AliExpress is a subsidiary of Alibaba which faces to the global market and it aims at building communication between small and medium enterprises and wholesalers. DHgate was established in 2004, helps Chinese SMEs to take advantage of cross-border e-commerce platforms to connect with the world market. In the condition of merchants settled, DHgate is more lenient than the AliExpress. In the logistics, DHgate supports more logistics ways, and it provides a more convenient online delivery service, simplified the sellers` logistics delivery processes. And AliExpress has advantage in withdrawing via RMB accounts, and DHgate has advantage in withdrawing via US dollars accounts. Besides, AliExpress can offer more marketing tools to the sellers, and the DHgate has more diverse marketing methods.
\end{abstract}

Keywords-AliExpress; DHgate; Comparative Analysis; B2C; Cross-border; E-commerce

\section{INTRODUCTION}

Cross border e-commerce is an international business that using the internet technology to build e-commerce platform for trading and payment, and distributing goods by cross-border logistics. In recent years, the cross-border e-commerce develops rapidly in China. The monitoring data from China Electronic Commerce Research Center shows that China cross-border e-commerce transactions amounted to 1.6 trillion yuan in 2011, 2012 is about 2 trillion yuan, 2013 exceeded 3.1 trillion yuan, and to 2014 first half of the year has reached 3 trillion, 2016 is expected to increase to 6.45 trillion yuan. The data from Commerce Department shows that more than 5000 crossborder business enterprises have arose in China.

There are two research methods used in this paper, one is the literature-search method. In order to make this paper more persuasive, we used multiple search engines to search the relevant data, so that we can collect the theory and data. The other method is comparative analysis. By comparing and analyzing the AliExpress and DHgate, we can get the difference between these two platforms.
In this paper, the researching-data is mainly from the AliExpress 's website (seller.aliexpress.com) and the DHgate`s website (www.dhgate.com).

\section{THE INTRODUCTION OF TWO PLATFORMS}

\section{A. The Intruduction of AliExpress}

The AliExpress, which is known as the "global taobao", was launched on April 2010. It aims at building communication between small and medium enterprises and wholesalers, carrying out the rapid sales of small lots and expanding the profit space. The AliExpress is an integrated international online trading platform with functions to order, payment, logistics, it is the only subsidiary of Alibaba which faces to the global market. The AliExpress services the overseas customers, it uses the alipay account to guarantee the transactions and the international logistics to deliver goods. It is the world's third largest online shopping platform.

In the early days of the AliExpress, it mainly focused on the small online foreign-trade-wholesale, its buyers were mostly foreign small businesses, which engaged in small wholesale purchasing and other small businesses. With the rapidly development of the AliExpress, the buyers crowed has changed, more and more individual buyers gathered to the AliExpress, replaced the small businesses and become the mainstream buyer groups. The $65 \%$ of the AliExpress' buyers are individual buyers, the rest are mainly engaged in small wholesale businesses. As a result, in order to satisfy the oversea individual buyers, the AliExpress announced that it has change into a global shopping platform from a small online foreign-tradewholesales platform in March 26, 2013.

As of March 2013, the range of AliExpress services has reached more than 220 countries and regions, covering apparel, home furnishing, 3C appliances, jewelry, and other 30 industry categories, its dominant industry are apparel, mobile communications, beauty health, shoes, bags, consumer electronics, jewelry, watches, home furnishing, computer, car and motorcycle accessories, lamps, etc. The merchandises which are in line with smaller, higher value-added, with a unique, prices are reasonable and the outside of the ban- restricted goods these conditions can be sole on AliExpress. More than 50 million overseas buyers visit the AliExpress in one day, its turnover sustained annual growth rate over $400 \%$, and the alexa ranking of AliExpress is 41 . 


\section{B. The Introduction of DHgate}

DHgate was established in 2004, is the world's leading online trading platform for foreign trades. DHgate is committed to helping Chinese's SMEs to take advantage of cross-border e-commerce platforms to the world market, opens up new international trade routes, makes online transactions become more simple, safe and efficient.

DHgate uses commission system, free registration fee, charged only after successful transaction between buyers and sellers. According to Paypal trading platform data, it shows that the online trade turnover of DHgate ranks the first in Asia-Pacific, it is the world's sixth-ranked ecommerce platform. At the same time, DHgate cooperates with foreign large enterprises actively, it is the largest customer of Paypal in Asia-Pacific, and it is the important strategic partner of Google and eBay in Chinese market as well. DHgate is also the business partner of UPS and the UPS`s services are embedded into DHgate`s platform.

DHgate announced the completion of several hundred million dollars financing in September 12 2014, the capital- injection institutions were CRE and TDF. After the capital injection, DHgate is going to expand in mobile business and emerging markets. DHgate has been built up a mature-technology cross-border electronic business platform, and accumulated global buyers through a wide range of overseas marketing methods. Now, after decades of development, the DHgate platform has 1.2 million registered domestic suppliers, 25 million kinds of online goods, services` range up to 224 countries and regions, with 5.5 million buyers, the platform produced an order every three seconds, the alexa ranking of DHgate is 1264 .

\section{THE COMPARATIVE ANALYSIS OF TWO PLATFORMS}

\section{A. Merchants Settled}

Merchants settled are divided into registration certification and industry settled two aspects. In registration certification, AliExpress register only via mailbox, it is necessary to use Alipay real name authentication after mail verification; DHgate requires a mobile phone number and email to register, it uses ID cards to process real-name authentication after the validation of cell phone number and email, after the first order confirmed by the platform it still needs to the bank certified. Since most businesses are settled from the domestic Taobao switch to cross-border e-commerce, they have real-name authentication Alipay, thus with respect to DHgate, the AliExpress registered certification is easier.

In industry settled, in order to further standardize the platform market, AliExpress and DHgate set settled rules for certain industries. AliExpress sets a strict standard on mobile phones, human hair, tablet PCs, memory cards these four categories, their technical service fee charged 150000 (conditional return).

DHgate is for hookah pipes, storage equipment, furs, jewelry, wedding boutique, celebrity dresses, brand mobile phones and sports goods which eight categories of assigned set the guidelines, but do not charge the annual fee. In contrast, the industry-settled conditions of the DHgate are more lenient than the AliExpress. However, the strict industry-settled conditions of the AliExpress create a more regulated market.

\section{B. Overseas Logistics}

AliExpress only support the sellers using the air logistics only. The logistics ways AliExpress supported include UPS, DHL, FedEx, TNT, EMS, SF, and China Post. Hong Kong Post Air Parcel Service. And it only authorized online shipping logistics tracking information as well as logistics tracking information provided by national postal official website, UPS official website, DHL official website, FedEx official website, TNT official website, TOLL official website, the official website of SF, EMS official website.

The logistics ways DHgate supported include EMS, UPS, DHL, FedEx, TNT, Ocean freight, China Post Air, China Post SAL, Hong Kong Post, TOLL, PARCEL FORCE, HERMES, Singapore post, DPD, TNT Post, ARAMEX, USPS, post link, Equick, DNJ, JILLION, JCEX, melodious Line (United Kingdom, Australia, Germany), European Business package, SF international, RPX, Ruston, XRU. DHgate also provides E mail treasure and DHLink these two more affordable prices, better quality services online logistics services. Sellers can deliver goods by applying online and delivering offline.

Both contrast, DHgate supports more logistics ways, and it provides more convenient online delivery services, simplified the sellers` logistics delivery processes.

\section{Payment And Transfer}

The sellers of AliExpress can set RMB accounts and US dollar accounts on the platform. After the buyers confirm receipt and agree to the lenders and logistics information displays delivery confirmation, the platform will transfer to the sellers` different accounts based on the payment methods of the buyers: when the buyers choose a credit card (RMB channel) or credit card (dollars channel), Western Union, MoneyBookers, Bank Transfer, Qiwi and other ways to make a payment, the payments will import into Alibaba guarantee accounts. After the transactions reach lending conditions, the money will transfer into the sellers`Alipay accounts (RMB accounts), or the sellers dollar accounts. Joining the AliExpress "special loan program" and enjoying special transferring service, the sellers' accounts can be transferred after delivery by the sellers and audited by AliExpress. In terms of fees, RMB accounts withdrawals do not charge fees, but the US dollar accounts need \$ 15 fee. Further international Alipay service for sellers to charge $5 \%$ of each order. The AliExpress provides credit services to good credit sellers, daily interest is $0.5 \%$.

In DHgate, the sellers can set RMB accounts and US dollar accounts as well. The difference is that, after the buyers confirm receipt and agree to the lenders and logistics information displays delivery confirmation, no matter what payment the buyers choose, the platform will transfer to the sellers DHgate virtual accounts in the form of dollar. The DHgate also provides advance transferring service for the application-accepted sellers, but only part of the amounts that get approved in advance, not all the money, and also need to charge $1-2 \%$ commission. In terms of withdrawals, the sellers can withdraw via RMB accounts and US dollar accounts, platform will exchange the US dollars of the RMB based on the current exchange rate when withdrawing by the RMB accounts (the specific exchange rate is subject to the current rate of funds 
credited into accounts), and it needs to charge the amount of $1 \%$ of the extract as a third party exchange fee; When withdrawing by the RMB accounts, when the virtual accounts 'balance is greater than equal to $\$ 5,000$, DHgate will bear the required handling charge; When the virtual accounts balance is less than $\$ 5,000$, DHgate requires the sellers to bear $\$ 15$ fee. DHgate gets commission from buyers rather than sellers: the quote above $\$ 200$, the commission ratio is $4.5 \%$; the quote below $\$ 200$, the commission ratio is $10-14 \%$. The DHgate also provides credit services to good credit sellers, daily interest is $0.417 \%$, and charge the $1 \%$ of the credit limit as the handling charge.

Both contrast, AliExpress has advantage in withdrawing via RMB accounts, and DHgate has advantage in withdrawing via US dollars accounts. In orders' commission, although AliExpress requires sellers charge $5 \%$ of the fee for each order, this fee will add to the prices of the goods finally and borne by the buyers. Therefore DHgate's fee is less when the orders' price is less than $\$ 200$, and when the orders ' price is more than $\$ 200$ the AliExpress fee is less. In the aspect of loans, AliExpress loans without fees, but its ratio is higher than DHgate.

\section{Marketing}

There are a lot of marketing tools that sellers can use in AliExpress. Except the limit period and discount, full set reduction, shopping coupon, shopping discount these selfmarketing tools, the AliExpress also offers E-mail marketing, affiliate marketing, AliExpress train, shop subscription and other marketing tools. AliExpress platform also organizes promotional activities and groupbuying activities to help increase the exposure and the sales of the sellers, such as SuperDeals, Weekend Deal, GaGa Deals, Russia Group Buying and other activities. The AliExpress also provides the sellers historical customers 'information statistical functions to facilitate the sellers' secondary marketing. AliExpress' data blocks provides business analysis functions to sellers, including the Real-Time -Storm, which can search the amount within 24 hours of exposure, views, number of visitors, number of pages per visit; The Shop-Overview, which can analyze the historical traffic and the retail transactions. The AliExpress also offers the analysis of the retail traffic sources function, the analysis of the store decoration trends function, the analysis of the commodity specific data function. Sellers can make appropriate marketing strategies for the products through the analysis of these detail data. The data blocks offers opportunities-discovery function as well, includes the analysis of various industries overview and the information of the blue sea industries. It also provides the products-chosen expert function that can analyze the hot-sell products and hot-search products, and the search-word analyzing function that can analyze the hot-search words, the burst words and the cold-search words. The opportunities-discovery function can provide data reference for the sellers when they settle into industries and selling products, help the sellers make decisions.

DHgate provides the sellers with the shopping activities (including discounts and price reduction directly) and coupon these self-marketing tools. Besides, DHgate also offers a series of marketing tools, such as the Google shopping marketing, products-traffic-train, fixed-price advertising, bidding advertising, exhibition plan, sunflower, DHgate international consortium, mail marketing, visual advertising, platform activity etc. DHgate helps the sellers with their secondary marketing through My Buyers block. DHgate provides sellers with the Data Intelligence Tools for data analyzing, including the shop overview function that can check the shops operating data, the industries overview function that can analyze the market information and mining the business opportunities, the search-words tracing function that can analyze that buyers' behaviors and buyers` information deeply. However, only the shop overview function is free to the buyers, the other functions need charge.

In contrast, AliExpress offers more marketing tools to the sellers, and the DHgate has more diverse marketing methods. The former provides the sellers with data analyzing tools for free, the latter needs charge.

\section{E. Customers Service}

In the aspect of customers ' service, both AliExpress and DHgate have the complete protocol $s$ and disputes handling system, helping settle the disputes between sellers and buyers: In the aspect of pre-delivery protocol, AliExpress allows the buyers cancel the orders after the orders paid successfully and before the sellers deliver the goods, it is more favorable for buyers. But the buyers of the DHgate just can cancel the orders after 24 hours they pay the orders successfully, it is more favorable for sellers. In the aspect of delivery protocol, if the sellers ' promisingdelivery-time is less than 5 days, buyers can apply for refunds as soon as the sellers deliver goods; if the sellers promising-delivery-time is no less than 5 days, buyers can apply for refunds after 5 days the sellers deliver goods. DHgate allows the buyers apply for refunds in 4-120 days after the sellers deliver the goods. Both of AliExpress and DHgate assure the rights of the buyers. In the aspect of platforms intervene disputes, under the circumstance of both of buyers and sellers cannot reach a consensus, the system will commit the disputes to the AliExpress to adjudicate 16 days after the dispute rose automatically, AliExpress will handle the disputes in 2 working days. But the DHgate disputes-automatic-commit-time is 11 days after the dispute rose, it is more efficient than the AliExpress.

In terms of customer communication channels, the AliExpress provides the station letter, e-mail and the instant chatting software TradeManager, the DHgate provides the station letter, e-mail and the DHgate Communication software. DHgate also can set subaccounts based on the main account, and give them different rights, so they can help the main account serve the customers better. In terms of the shop credit rating system, the credit level of AliExpress is divided into 15 levels, each level is clearly represented by the rating signs combined by medal, diamond or crown. The credit level of DHgate is divided into 4 levels: top, perfect, standard and lower-standard, only the top and the perfect level has the appropriate sign. Therefore the AliExpress has more complete credit system. In addition, DHgate has the Foreign Buy block, it can display the requests of the customers and connect the customers with the sellers. 


\section{CONCLUSIONS}

Cross-border e-commerce develops rapidly at the stimulating of domestic and international economic environment and the supports of the policies. In the various forms of the cross-border e-commerce, B2C, the form that connects the enterprises and buyers directly, has a great potential. Cross-border e-commerce platforms, as the bridges of the sellers and the buyers, they play very important roles. This paper takes two of the country's biggest $\mathrm{B} 2 \mathrm{C}$ cross-border e-commerce platforms as research objects, uses the method of comparative analysis to analyze them from 5 aspects: merchants settled, overseas logistics, payment loans, marketing and customer service. Because of the limit of the information, the research has the inadequate in depth study.

\section{REFERENCES}

[1] Fan Xiaoyun, Comparison Analysis and Selection of the Mode of the Electric Business Platform of the Cross-border Trade in China, Foreign Economic Relations and Trade, Vol 02,2015,pp. 12-14.

[2] Zhang Xuewei, Innovational Business Model Analysis of "AiMeiGou" International E-commercial Platform, East China University of Science and Technology, 2015,pp.20-22.
[3] Chenggang $\mathrm{Mu}$, The Research of Cross-border E-commerce Models in China, Shanghai Academy of Social Science, 2014,pp33-35.

[4] Zhang Rui, SWOT Analysis and Countermeasures for the Development of Cross-border E-commerce, Journal of Shandong Institute of Business and Technology, Vol 03,2015,pp.88-93.

[5] Zhang Xuecheng, Electronic Practice of International Trade, Hangzhou: Zhejiang University Press, 2010,pp.55-56.

[6] Wang Wailian, Liu Shuzhen, The Analysis of The Current Situation And Suggestions of The Cross-border E-commerce in China, E-Business Journal, Vol 09, 2013,pp.23-24, doi :10.14011/j.cnki.dzsw.2013.09.024.

[7] Huang Hailong, The Research of The Internet Finance Based on The E-commerce Platforms, Shanghai Finance, Vol 08, 2013, pp18-23.

[8] Zhang Baoming, Zhou Peifeng, Meng Ling, The Research of The E-commerce And Logistics development, Logistics Sci-Tech, Vol10, 2014,pp.54-58,doi :10.13714/j.cnki.1002-3100.2014.10.017

[9] Ji Fang, Zhang Xiaheng, The Model Creation And The Develop Tendency of The Logistics in Cross-border E-commerce, China Business and Market, Vol 06,2015,pp.14-20,doi: 10.14089/j.cnki.cn11-3664/f.2015.06.003

[10] Wang Jiayan, The development Research of The Interaction of Cross-border And Logistics, The Brand, Vol 05, 2014,pp.105-10 